\title{
Association of Erectile Dysfunction and Hypogonadism with Metabolic Syndrome in Men with Lower Urinary Tract Symptoms of Younger and Older Age Groups
}

\author{
Elena Novikova1,2, Vera Selyatitskaya1, Igor Mitrofanov¹, Boris Pinkhasov1,2, \\ Ani Karapetyan ${ }^{1,2}$ \\ ${ }^{1}$ Siberian Branch of Russian Academy Medical Sciences, Scientific Centre of Clinical and Experimental Medicine, \\ Novosibirsk, Russia \\ ${ }^{2}$ Department of Urology, Regional Medical and Diagnostic Center, Novosibirsk, Russia \\ Email: rmdc.nsk@gmail.com, ccem@centercem.ru, mim@centercem.ru, pin@centercem.ru, \\ docani1987@gmail.com
}

Received 22 July 2014; revised 22 August 2014; accepted 10 September 2014

Copyright (C) 2014 by authors and Scientific Research Publishing Inc.

This work is licensed under the Creative Commons Attribution International License (CC BY).

http://creativecommons.org/licenses/by/4.0/

c) (i) Open Access

\section{Abstract}

The aim of this study was to evaluate the incidence and severity of erectile dysfunction (ED) and hypogonadism in 193 men presenting with lower urinary tract symptoms (LUTSs) in the younger (20 39 years old) and older (40 - 60 years old) age groups depending on the presence of metabolic syndrome (MetS). Triglycerides, glucose and high-density lipoprotein cholesterol, serum total testosterone (TT), sex hormone-binding globulin, prostate-specific antigen, pituitary hormones levels were measured in serum. Standardized criteria (2009) were used to determine the prevalence of MetS. Patients were assessed based on the International Prostate Symptom Score (IPSS) and the IPSS-Quality of Life (IPSS-QoL) for LUTSs and the International Index of Erectile Function (IIEF) for ED. Hypogonadism was determined in accordance with the ISA, ISSAM, EAU, EAA and ASA recommendations. In men with MetS in the younger age group the incidence of ED was 2.4 times higher, and that of low TT level and hypogonadism was 8.4 times higher compared to men without the syndrome. In the older age group, an increased incidence of ED and low TT level in men with MetS compared to men without the syndrome was on the trend level, but the incidence of hypogonadism was for sure higher (by 1.6 times). In conclusion, the study showed that ED and hypogonadism are associated with MetS by a high degree of certainty in young men with LUTSs. In men of the older age group with LUTSs, the presence of MetS is not so clearly associated with ED and hypogonadism due to the fact that the incidence of these urogenital diseases is also high in men without MetS. 


\section{Keywords}

\section{Erectile Dysfunction, Testosterone, Hypogonadism, Metabolic Syndrome, Men of Younger and Older Age Groups, Lower Urinary Tract Symptoms}

\section{Introduction}

In most countries, the representation of older age groups is increasing both among women and men. Increasing age is associated with the extinction of the reproductive function and the accumulation of chronic diseases such as cardiovascular diseases, atherosclerosis, obesity, metabolic syndrome (MetS), type 2 diabetes, urogenital diseases, etc. Lower urinary tract symptoms (LUTSs), erectile dysfunction (ED) and late-onset hypogonadism $(\mathrm{LOH})$ are the most common urogenital diseases in aging men [1] [2], and their prevalence is significantly increasing among elderly and old men [1] [3]-[6]. The age-related androgen deficiency accompanied by low testosterone level, which has a critical role in the maintenance of homeostatic and morphologic corpus cavernosum components essential for normal erectile physiology, is very significant for the mechanisms of ED pathogenesis [7]. Low blood testosterone level is also the main mechanism of LOH development [6]. As for LUTSs, it has been shown that absolute sex steroid hormone (testosterone and estradiol) levels and the rates at which the levels change may be important in the development of urologic conditions in aging men [8]. Consequently, the agerelated androgen deficiency plays an important role in the development of numerous urogenital diseases in aging men.

Androgen deficiency is a multisystem syndrome whose clinical presentation depends on the age and other patient-specific characteristics such as comorbidities, genetic, environmental, and sociocultural factors [9]. Androgen deficiency is associated with the development of obesity and MetS and is an independent risk factor for cardiovascular diseases. Evidence that whole-body insulin sensitivity is reduced in testosterone deficiency and increases with testosterone replacement supports a key role of this hormone in glucose and lipid metabolism [10]. In turn, MetS and its typical etabolic disorders are also considered as risk factors for urological diseases and erectiledys function in men [11] [12].

The relationship of MetS with ED, LOH and LUTSs is usually studied by examining elderly and old men, which is quite natural as all these diseases mainly occur and accumulate with increasing age [1] [2] [12]. However, in recent years, the prevalence of MetS significantly increased among young men, including children and adolescents [13] [14], which is thought to be caused by lifestyle change, reduced physical activity, consumption of high-energy foods and drinks, sleep disorders, etc. [15]. It is unknown to what extent MetS will influence the occurrence of the above urogenital diseases in young men.

We conducted a comparative study of the incidence and severity of erectile dysfunction and hypogonadism in men presenting with LUTSs in the younger (20 - 39 years old) and older (40 - 60 years old) age groups depending on the presence of metabolic syndrome.

\section{Patients and Methods}

\subsection{Patients}

193 consecutive patients aged 20 to 60 years old who consulted with an urologist at the Regional Medical and Diagnostic Center about complaints of voiding dysfunction were included. All patients gave their informed written consent prior to their inclusion in the study. The study was approved by the local Institutional Ethics Committee. Patients with genital abnormalities, systemic diseases such as diabetes mellitus, thyroid diseases or neurological diseases or a history of transurethral resection or pelvic surgery and patients taking medication for LUTSs or ED were excluded. The patients were divided into two groups according to the age: the younger (20 39 years old) and the older (40 - 60 years old) age groups.

\subsection{Patient Evaluation}

After taking a detailed medical history and performing a digital rectal examination, waist circumference (cm) was determined using measuring tape to the nearest $5 \mathrm{~mm}$. Blood samples were drawn between 8:00 and 10:00 
a.m. from overnight-fasting patients. Triglycerides, glucose and high-density lipoprotein (HDL) cholesterol levels were measured in serum on the biochemical analyzer Roche Diagnostics/Hitachi 912. Serum Total Testosterone (TT), Sex Hormone-Binding Globulin (SHBG), Prostate-Specific Antigen (PSA), prolactin, FollicleStimulating Hormone (FSH), Luteinizing Hormone (LH), Thyroid-Stimulating Hormone (TSH) were measured with the IMMULITE 2000 immunoassay system (Siemens Healthcare Global). The calculation of free testosterone level was carried out using the Free \& Bioavailable Testosterone calculator on the ISSAM site (http://www.issam.ch/freetesto.htm).

\subsection{MetS Assessment}

Standardized criteria were used to determine the prevalence of MetS and its individual components among the examined men [16]. The values of waist circumference $>102 \mathrm{~cm}$ were used to diagnose abdominal obesity. Categorical cut points for revelation of hypertriglyceridemia (elevated triglycerides $\geq 1.7 \mathrm{mmol} / \mathrm{L}$ or drug treatment for elevated triglycerides is an alternate indicator), reduced HDL-cholesterol $(<1.0 \mathrm{mmol} / \mathrm{L}$ or drug treatment for reduced HDL-C is an alternate indicator), elevated blood pressure (systolic $\geq 130$ and/or diastolic $\geq 85 \mathrm{~mm} \mathrm{Hg}$, or antihypertensive drug treatment in a patient with a history of hypertension is an alternate indicator), elevated fasting glucose (blood glucose $\geq 5.6 \mathrm{mmol} / \mathrm{L}$, or drug treatment of elevated glucose is an alternate indicator) were used. MetS was diagnosed when at least three of any components were present.

\subsection{LUTSs and ED Assessment}

Validated questionnaires were used for this investigation. Patients were assessed based on the International Prostate Symptom Score (IPSS) and the IPSS-Quality of Life (IPSS-QoL) for LUTSs [17] and the International Index of Erectile Function (IIEF) for ED [18]. LUTSs were classified as mild (IPSS of $\leq 7$ ), moderate (IPSS of 8 - 19) or severe (IPSS of 20 or more). The severity of ED according to the IIEF score was classified into four categories as severe (1 - 10), moderate (11 - 16), mild (17 - 25) or no ED (26 - 30).

\subsection{Hypogonadism Assessment}

Hypogonadism is a clinical condition comprising both symptoms and biochemical evidence of testosterone deficiency. In accordance with the ISA, ISSAM, EAU, EAA and ASA recommendations [6], the manifestations of hypogonadism include: low libido, ED, decreased muscle mass and strength, increased body fat, decreased bone mineral density and osteoporosis, decreased vitality and depressed mood. None of these symptoms are specific to the low androgen state but may raise suspicion of testosterone deficiency. One or more of these symptoms must be corroborated with a low serum testosterone level [6]. In accordance with the purpose of research, we chose ED as such a symptom. The lower limit of normal serum TT level was $12 \mathrm{nmol} / \mathrm{L}$ [19]. Patients were also assessed based on the Aging Males' Symptoms (AMS) scale for ageing male symptoms [20].

\subsection{Statistical Analysis}

Statistical data processing was performed using the STATISTICA program, v. 10.0 (Stat Soft Inc., USA). The results are presented as incidence in \%, the comparative analysis was performed using the Pearson's $\chi^{2}$ test (for two groups with Yates correction), and as mean indicator values and the standard deviation ( $M \pm S D$ ); the comparative analysis was performed using the Kruskal-Wallis test with multiple comparisons of mean ranks and the Mann-Whitney test for paired comparisons. The relationships between ED and other studied variables were estimated by the Spearman Rank Correlation coefficient. The minimum probability of the null hypothesis validity was accepted at the $5 \%$ significance level $(p<0.05)$.

\section{Results}

A total of 193 patients who consulted with an urologist about complaints of voiding dysfunction were examined. To determine the age characteristics of the studied parameters, the men were divided into two age groups: the younger one aged 20 to 39 years old, which included 84 men, and the older one aged 40 - 60 years old, which included 109 men; the average age in the groups was $30.1 \pm 5.6$ and $53.8 \pm 5.7(p<0.0001)$, respectively. To exclude cases of prostate tumors, all patients underwent a blood test for PSA; its serum level in men of the younger age group was $0.94 \pm 0.77$, while in the older group it was $1.82 \pm 2.06(p=0.0210)$. To assess the severity of 
LUTS, the patients were surveyed using IPSS questionnaires. In the younger age group, the total of the IPSS domain scores was $2.3 \pm 3.9$ points, and in the older age group it was $9.9 \pm 5.9$ points $(p=0.0048)$. Consequently, LUTS severity can be characterized as mild in the younger age group and as moderate in the older age group. In the younger age group, the IPSS-QoL domain scores was $1.4 \pm 1.6$ points, and in the older age group it was $3.6 \pm 1.8$ points $(p=0.0008)$, which is consistent with a greater severity of LUTS symptoms in men of the older age group. No cases of severe LUTS were revealed in men of both the older and the younger age group.

Table 1 presents the general characteristic of the studied indices in men of different age groups. On the whole, men of the older age group had worse indices than men of the younger age group except the incidence of abdominal obesity in the group.

To investigate the role of MetS in the development of urogenital disorders in each age group, the examined men were divided into two subgroups: those with MetS and those without MetS. The ratio of the numbers of men with and without MetS in the younger age group was 1:3.4, while in the older age group it was 1:1.3. In the younger age group, the average age of men with MetS was $32.9 \pm 4.7$, and that of men without MetS was $29.8 \pm$ 5.6 years $(p>0.05)$, while in the older age group it was $53.7 \pm 3.0$ and $53.6 \pm 5.6(p>0.05)$, respectively.

Table 2 presents the results of determining TT and SHBG levels and calculating free testosterone levels in sera of men of different age groups depending on the presence of MetS. In the younger age group, TT and free

Table 1. General characteristics of men in two age groups.

\begin{tabular}{|c|c|c|c|}
\hline Index & Group 1 (20 - 39.9 years old) $N=84$ & Group 2 (40 - 60 years old) N = 109 & $p$ \\
\hline Average age, years $(\mathrm{m} \pm \mathrm{SD})$ & $30.1 \pm 5.6$ & $53.8 \pm 5.7$ & $<0.0001$ \\
\hline Body mass index, $\mathrm{kg} / \mathrm{m}^{2}(\mathrm{~m} \pm \mathrm{SD})$ & $26.9 \pm 5.1$ & $28.7 \pm 3.9$ & 0.0105 \\
\hline Androgen deficiency by AMS, n (\%) & $34(40 \%)$ & $96(88 \%)$ & $<0.0001$ \\
\hline Erectile dysfunction, n (\%) & $29(35 \%)$ & $75(69 \%)$ & $<0.0001$ \\
\hline Total testosterone level < $12 \mathrm{nmol} / \mathrm{L}, \mathrm{n}(\%)$ & $11(13 \%)$ & $49(45 \%)$ & 0.0016 \\
\hline Hypogonadism, n (\%) & $11(13 \%)$ & $43(39 \%)$ & $<0.0001$ \\
\hline Metabolic syndrome, n (\%) & $19(23 \%)$ & $47(43 \%)$ & $<0.0083$ \\
\hline Abdominal obesity, n (\%) & $18(21 \%)$ & $26(24 \%)$ & 0.7306 \\
\hline Arterial hypertension, n (\%) & $34(40 \%)$ & $88(81 \%)$ & $<0.0001$ \\
\hline Hyperglycemia, n (\%) & $15(18 \%)$ & $47(43 \%)$ & 0.0003 \\
\hline Hypertriglyceridemia, n (\%) & $26(31 \%)$ & $51(47 \%)$ & 0.0350 \\
\hline Low level of HDL cholesterol, n (\%) & $22(16 \%)$ & $54(50 \%)$ & 0.0030 \\
\hline
\end{tabular}

Table 2. Hormone levels in the blood serum of men in two age groups depending on the presence of metabolic syndrome (M $\pm \mathrm{SD})$.

\begin{tabular}{|c|c|c|c|c|c|}
\hline \multirow{3}{*}{ Indices } & \multicolumn{2}{|c|}{ Group 1 (20 - 39.9 years old) } & \multicolumn{2}{|c|}{ Group 2 (20 - 39.9 years old) } & \multirow{3}{*}{$p$} \\
\hline & $-\mathrm{MC} \mathrm{N}=65$ & $+\mathrm{MC} \mathrm{N}=19$ & $-\mathrm{MC} \mathrm{N}=62$ & $+\mathrm{MC} \mathrm{N}=47$ & \\
\hline & 1 & 2 & 3 & 4 & \\
\hline Total testosterone level, nmol/L & $20.7 \pm 7.9$ & $10.5 \pm 3.1$ & $14.5 \pm 5.8$ & $10.9 \pm 4.2$ & $\begin{array}{l}1-2<0.0001 \\
1-3<0.0001 \\
3-4=0.0007\end{array}$ \\
\hline Sex hormone binding globulin, nmol/L & $36.6 \pm 16.5$ & $22.7 \pm 16.4$ & $37.8 \pm 15.8$ & $38.9 \pm 22.5$ & $\begin{array}{l}1-2=0.0282 \\
2-4=0.0488\end{array}$ \\
\hline Free testosterone, pmol/L & $424 \pm 215$ & $253 \pm 80$ & $274 \pm 158$ & $196 \pm 95$ & $\begin{array}{l}1-2=0.0025 \\
1-3=0.0120 \\
3-4=0.0330\end{array}$ \\
\hline Prolactin, IU/L & $7.60 \pm 3.36$ & $8.47 \pm 3.08$ & $8.13 \pm 3.02$ & $9.11 \pm 4.28$ & \\
\hline Follicle-stimulating hormone, IU/L & $3.61 \pm 2.96$ & $3.42 \pm 1.65$ & $3.29 \pm 1.67$ & $2.98 \pm 1.97$ & \\
\hline Luteinizing hormone, IU/L & $3.51 \pm 1.79$ & $3.27 \pm 1.85$ & $3.81 \pm 2.02$ & $4.07 \pm 3.66$ & \\
\hline Thyroid-stimulating hormone, IU/L & $1.66 \pm 0.79$ & $1.71 \pm 0.97$ & $1.63 \pm 0.71$ & $1.69 \pm 0.95$ & \\
\hline
\end{tabular}


testosterone levels in serum was almost 2 times lower in men with MetS compared to men without MetS. In the older age group, the presence of MetS was also associated with decreased levels of TT and free testosterone in blood, but it was less pronounced than in men of the younger age group and was mainly due to decreased levels of total and free testosterone in men without metabolic syndrome. SHBG level in blood was 1.5 times lower in men with MetS in the younger age group, which was not found in men of the older age group. The absence of differences in FSH and LH levels in serum suggests that changes in TT levels in men with MetS are not associated with changes in the secretion of pituitary hormones. The absence of changes in prolactin and TSH levels in serum allows us to exclude the presence of other endocrine pathologies such as thyroid dysfunction or hyperprolactinemia contributing to the formation of androgen deficiency in the organism.

Table 3 presents the results of measuring the incidence of urogenital disorders in men of different age groups depending on the presence of MetS. The incidence of symptoms of androgen deficiency by AMS was higher in both subgroups of older men compared to younger men in respective subgroups. But in both age groups, the presence of MetS was not significantly associated with an increased incidence of symptoms of androgen deficiency by AMS; this relationship was observed only on the trend level. In contrast to the AMS, the incidence of ED, low TT level in blood and hypogonadism were significantly higher in men with MetS in the younger age group: the incidence of ED was 2.4 times higher, and that of low TT level and hypogonadism was 8.4 times higher compared to men without the syndrome. In the older age group, an increased incidence of ED and low TT level in men with MetS compared to men without the syndrome was on the trend level, but the incidence of hypogonadism was significantly higher (by 1.6 times).

Table 4 presents the results of correlation analysis of the relationships between the investigated indices and the presence of ED in men of different age groups. Significant negative correlations were revealed between the presence of ED and testosterone levels in blood of men of the younger and the older age groups. Correlations were also revealed in the younger age group between the presence of ED and indices indicating the development of MetS in men. These were positive correlations with body weight and waist circumference, blood pressure and indices characterizing disorders of carbohydrate and fat metabolism. In the older age group, significant positive correlations were found only between ED and blood pressure.

\section{Discussion}

The study revealed a significant increase in the incidence of urogenital disorders in men with LUTS as well as the incidence of MetS and its components with increasing age, which is consistent with literature data [1] [3]-[6]

Table 3. Characteristics of men in two age groups depending on the presence of metabolic syndrome.

\begin{tabular}{|c|c|c|c|c|c|}
\hline \multirow{3}{*}{ Indices } & \multicolumn{2}{|c|}{ Group 1} & \multicolumn{2}{|c|}{ Group 2} & \multirow{3}{*}{$p$} \\
\hline & $-\mathrm{MC} \mathrm{N}=65$ & $+\mathrm{MC} \mathrm{N}=19$ & $-\mathrm{MC} \mathrm{N}=62$ & $+\mathrm{MC} \mathrm{N}=47$ & \\
\hline & 1 & 2 & 3 & 4 & \\
\hline Average age, years $(\mathrm{M} \pm \mathrm{SD})$ & $29.8 \pm 5.6$ & $32.9 \pm 4.7$ & $53.6 \pm 5.6$ & $53.7 \pm 3.0$ & $\begin{array}{l}1-3<0.0001 \\
1-4<0.0001 \\
2-3<0.0001 \\
2-4<0.0001\end{array}$ \\
\hline Androgen deficiency by AMS, n (\%) & $23(35 \%)$ & $11(58 \%)$ & $53(85 \%)$ & $43(91 \%)$ & $\begin{array}{l}1-3<0.0001 \\
2-4=0.0016\end{array}$ \\
\hline Erectile dysfunction, n (\%) & $17(26 \%)$ & $12(63 \%)$ & 38 (61\%) & 37 (79\%) & $\begin{array}{l}1-2=0.0005 \\
1-3<0.0001\end{array}$ \\
\hline Total testosterone $<12 \mathrm{nmol} / \mathrm{L}, \mathrm{n}(\%)$ & $3(5 \%)$ & $8(42 \%)$ & $23(37 \%)$ & $26(55 \%)$ & $\begin{array}{l}1-2=0.0001 \\
1-3=0.0156 \\
1-4<0.0001\end{array}$ \\
\hline Hypogonadism (\%) & $3(5 \%)$ & $8(42 \%)$ & 19 (31\%) & $24(51 \%)$ & $\begin{array}{l}1-2=0.0009 \\
1-3=0.0006 \\
3-4=0.0288\end{array}$ \\
\hline
\end{tabular}


Table 4. Correlation analysis results of the relationships between the studied variables and the presence of erectile dysfunction in two age groups of men.

\begin{tabular}{ccccc}
\hline Indices & & Group 1 & & Group 2 \\
Body mass & $\mathrm{r}_{\mathrm{s}}$ & $p$ & $\mathrm{r}_{\mathrm{s}}$ & $p$ \\
\cline { 2 - 4 } Body mass index & 0.44 & 0.0006 & -0.05 & 0.6836 \\
Waist circumference & 0.38 & 0.0009 & 0.07 & 0.4912 \\
Systolic blood pressure & 0.37 & 0.0014 & 0.15 & 0.0355 \\
Diastolic blood pressure & 0.47 & $<0.0001$ & 0.22 & 0.0175 \\
Glucose & 0.47 & $<0.0001$ & 0.24 & 0.0975 \\
Triglycerides & 0.43 & 0.0002 & 0.17 & 0.2688 \\
Total cholesterol & 0.26 & 0.0329 & -0.12 & 0.3278 \\
HDL cholesterol & 0.23 & 0.0473 & 0.10 & 0.1524 \\
Testosterone & -0.35 & 0.0027 & -0.15 & 0.0002 \\
SHBG & -0.40 & 0.0005 & -0.38 & 0.8277 \\
Prolactin & -0.18 & 0.2490 & 0.04 & 0.6203 \\
Thyroid-stimulating hormone & 0.19 & 0.1025 & 0.05 & 0.2930 \\
Follicle-stimulating hormone & -0.07 & 0.5551 & 0.12 & 0.9458 \\
\hline
\end{tabular}

[21]. Our results on the occurrence of MetS and ED in men over the age of 40 years old having LUTS are consistent with other researchers' results. For instance, in [22] it was found that $39.9 \%$ of urologic patients aged 40 70 years old had MetS, while among patients with or without MetS the occurrence of ED was $79 \%$ and $61.9 \%$. For comparison, similar results in patients examined by us were $43 \%, 79 \%$ and $61 \%$, respectively (Table 1 and Table 3).

The only MetS component whose incidence slightly increased in the older age group as compared to this index value in the younger age group (24\% and $21 \%$, respectively) was abdominal obesity. This index was determined according to standardized criteria (16) by waist circumference $>102 \mathrm{~cm}$.

We have previously shown that abdominal (upper) obesity has more pronounced negative effects on human health than gynoid (lower) obesity [23], not only in women but also in men. The upper type of fat distribution is associated with intra-abdominal visceral fat accumulation, which plays an important role in the development of diabetes, lipid disorder, hypertension and atherosclerosis [24]. High incidence of abdominal obesity in the group of young men may be due to reduced physical activity and consumption of high-calorie foods, which is characteristic of lifestyle preferred by modern youth [25] [26]. Such lifestyle contributes to the accumulation of excess fat mass and earlier formation of obesity. Our results indicating that more than $20 \%$ of young men aged 20 to 39 years old inclusive have abdominal obesity suggest that they are at high risk for chronic noncommunicable diseases more typical of older age groups.

The presence of MetS in men of both age groups was associated with increased incidence of low TT level (less than $12 \mathrm{nmol} / \mathrm{L}$ ). In the younger age group, the incidence of low TT level was more than 8 times higher in men with MetS compared to men without MetS, while in the older age group this difference was 1.5 times. This may be due to the fact that in men of the older age group without MetS the incidence of low TT level was significantly higher, and in the presence of MetS it insignificantly changed compared to men of the younger age group, with or without MetS.

In this study, we used the value less than $12 \mathrm{nmol} / \mathrm{L}$ as a threshold of low TT level, without distinguishing between men with over thypogonadism (total testosterone $<8 \mathrm{nmol} / \mathrm{L}$ ) and borderline hypogonadism (total testosterone of 8 - $12 \mathrm{nmol} / \mathrm{L}$ ) [19]. In accordance with ISA, ISSAM, EAU, EAA and ASA recommendations [6], 
based on the data of younger men, there is a consensus that patients with serum total testosterone levels below 8 $\mathrm{nmol} / \mathrm{L}$ (230 ng per100 ml) will usually benefit from testosterone treatment. In our study, we did not aim to select men for testosterone treatment, that's why we did not distinguish between overt and borderline hypogonadism.

In men of the younger age group, regardless of the presence of MetS, the incidence of hypogonadism coincided with that of low TT level. In men of the older age group, the incidence of hypogonadism was slightly lower than that of low testosterone level. This may be due to the fact that we took into account only ED as a symptom of androgen deficiency, at the same time, there are other symptoms such as low libido, decreased muscle mass and strength, increased body fat, decreased bone mineral density and osteoporosis, decreased vitality and depressed mood [6] that we did not take into account. In men of both the younger and the older age group the incidences of ED were higher than those of hypogonadism. It can be supposed that a higher incidence of ED than that of hypogonadism may be due to the fact that we included men with LUTS in the study. In [2] it was shown that LUTS severity was an age-independent risk factor for ED and symptomatic late-onset hypogonadism.

In men of the younger age group with MetS SHBG level in blood was 1.5 times lower compared to men without MetS in the same age group. No decrease in SHBG level was observed in men with MetS in the older age group. It can be supposed that the reduction in the level of this sex hormone binding protein in blood of young men with MetS is a compensatory reaction aimed at increasing the relative amount of free physiologically active sex hormone testosterone in the formation of their hypogonadism. However, this may intensify the metabolism of free testosterone, which, in turn, may worsen androgen deficiency. In [27] it was shown that TT and SHBG levels were strongly associated with MetS and surrogate measures of insulin resistance. The authors of this paper hypothesized that since MetS and insulin resistance are risk factors for cardiovascular diseases and type 2 diabetes, early detection of men with decreased TT and SHBG levels in clinical settings will be beneficial for preventing future cardiovascular outcomes [27].

In men of the younger age group, despite a significantly higher incidence of low TT level and hypogonadism among men with MetS, there was no significant difference between men with or without MetS in the incidence of androgen deficiency detected by the AMS scale. These results are consistent with the opinion of experts whose recommendations [6] indicated that questionnaires such as Aging Male Symptom Score (AMS) and Androgen Deficiency in Aging Men (ADAM) are not recommended for the diagnosis of hypogonadism because of low specificity.

Our findings showed that the impact of MetS on the development of urogenital disorders more clearly manifests itself in young men. It is known that in older men sexual dysfunction is associated to a greater extent with testosterone deficiency and accumulation of chronic diseases (first of all, cardiovascular and endocrine ones), which have an adverse effect on the urogenital system [28] [29]. Accumulation of chronic diseases in older men makes it difficult to identify the impact of MetS on sexual dysfunction, because MetS itself is a cluster of risk factors for the diseases that are already present in older men [30]. Since the incidence of such diseases in young men is lower, the impact of MetS on the development of their sexual dysfunction is more pronounced. Importantly, in young men, obesity and MetS syndrome become risk factors for not only cardiovascular diseases, metabolic disorders and diabetes type 2, but also for sexual dysfunction. Therefore, measures to combat obesity and metabolic syndrome come to the fore in the prevention of early sexual dysfunction that affects the health of the family and society in general, have a negative impact on quality of life, promote physical and emotional dissatisfaction [31]. This assumption is confirmed by the results of the correlation analysis carried out by us. It was found that in men of the younger age group the presence of ED was significantly associated with body weight and MetS components. In the older age group, the presence of ED was significantly associated only with blood pressure, which is indicative of the important role of the vascular component in its development. The statement of the need for weight reduction in treating sexual dysfunction is consistent with other researchers' opinions [32]. In [33] the authors note that successful weight loss has many other health benefits and should be first priority. Testosterone therapy has the potential to augment diet-induced weight loss and may have additional benefits on other, androgen-responsive tissues beyond its effects on fat mass.

In the course of evolution, the mechanisms of body fat accumulation developed and were preserved as a way of survival in extreme environments. However, under conditions of excess food and insufficient physical activity these previously positive mechanisms become the leading element of accumulation of negative changes in the organism. Hypogonadism diagnosed in young men with MetS has symptoms of age-related hypogonadism typi- 
cal of elderly men. This suggests that the development of MetS and hypogonadism in young men is associated with accelerated aging processes. Our hypothesis is consistent with data from scientific literature. For instance, in the literature review [34], the authors come to a conclusion that hypogonadism, ED, obesity, insulin resistance and MetS often coexist in the same subjects. This cluster of abnormalities is associated with an increased risk for diabetes and cardiovascular diseases, affecting not only quality of life but also life expectancy.

The question arises as to what develops in young men as the initial element of the studied comorbidpathology-MetS with abdominal obesity or hypogonadism? Different opinions were expressed in discussing the results of studies conducted with the involvement of men aged over 40 years old. For instance, in the literature review [35], the authors suggest that overweight or chronic illness might explain late-onset hypogonadism and low TT level. In this regard, they conclude that in this situation it would seem most logical to begin treatment with lifestyle modification and weight reduction, and to optimize the treatment of comorbidities, before testosterone supplementation, which is expensive and of uncertain benefit. In the literature review [36], the authors discuss individual components of the mutual influence of androgen deficiency and MetS without singling out the initial element of this relationship. Our study describes the phenomenon of association of MetS with low testosterone level and hypogonadism in young men under the age of 40 years old. Currently, there is a large flow of information on high incidence of obesity and MetS in adolescents, decreased physical activity due to watching TV and playing computer games characteristic of modern young men, the consumption of large amounts of carbohydrates and saturated fats with tonics, crisps and other substitutes of the "traditional" diet. Summing up the above facts, it can be supposed that, in contrast to aging men, in young men the initial element of the relationship between MetS and hypogonadism is MetS, in particular, abdominal obesity. However, verification of this hypothesis requires further research.

\section{Conclusion}

The study showed that ED and hypogonadism are associated with MetS with a high degree of certainty in young men with LUTSs in the age group 20 to 39 years old inclusive. In men of the older age group with LUTSs (40 60 years old) the presence of MetS is not so clearly associated with ED and hypogonadism due to the fact that the incidence of these urogenital diseases is also high in men without MetS. Our findings suggest that increasing prevalence of abdominal obesity and MetS in young men, which is to a great extent caused by a sedentary lifestyle and consuming foods rich in carbohydrates and saturated fats, becomes a risk factor not only for cardiovascular diseases and diabetes type 2 but also for urogenital diseases including hypogonadism and ED. Given that the incidence of MetS in men of the younger age group is $23 \%$, it can be supposed that the incidence of ED among young men is already rather high and will increase following the increase in the incidence of obesity, which can create a lot of not only medical but also social problems.

\section{Acknowledgements}

The authors would like to acknowledge of Scientific Centre of Clinical and Experimental Medicine director prof. Vyacheslav A. Shkurupiy for support of the study.

\section{References}

[1] McVary, K. (2006) Lover Urinary Tract Symptoms and Sexual Dysfunction: Epidemiology and Pathophysiology. BJU International, 97, 23-28. http://dx.doi.org/10.1111/j.1464-410X.2006.06102.x

[2] Bozkurt, O., Bolat, D., Demir, O., Ucer, O., Sahin, A., Ozcift, B., Pektas, A., Turan, T., Gumus, B.H., Can, E., Bolukbasi, A., Erol, H. and Esen, A. (2013) Erectile Function and Late-Onset Hypogonadism Symptoms Related to Lower Urinary Tract Symptom Severity in Elderly Men. Asian Journal of Andrology, 15, 785-789. http://dx.doi.org/10.1038/aja.2013.44

[3] Corona, G., Lee, D.M., Forti, G., O’Connor, D.B., Maggi, M., O’Neill, T.W., Pendleton, N., Bartfai, G., Boonen, S., Casanueva, F.F., Finn, J.D., Giwercman, A., Han, T.S., Huhtaniemi, I.T., Kula, K., Lean, M.E.J., Punab, M., Siman, A.J., Vanderschueren, D., Wu, F.C.W. and Group, E.S. (2010) Age-Related Changes in General and Sexual Health in Middle-Aged and Older Men: Results from the European Male Ageing Study (EMAS). The Journal of Sexual Medicine, 7, 1362-1380. http://dx.doi.org/10.1111/j.1743-6109.2009.01601.x

[4] Selvin, E., Burnett, A.L. and Platz, E.A. (2007) Prevalence and Risk Factors for Erectile Dysfunction in the US. The American Journal of Medicine, 120, 151-157. http://dx.doi.org/10.1016/j.amjmed.2006.06.010 
[5] Kubin, M., Wagner, G. and Fugl-Meyer, A.R. (2003) Epidemiology of Erectile Dysfunction. International Journal of Impotence Research, 15, 63-71. http://dx.doi.org/10.1038/sj.ijir.3900949

[6] Wang, C., Nieschlag, E., Swerdloff, R., Behre, H.M., Hellstrom, W.J., Gooren, L.J., Kaufman, J.M., Legros, J.-J., Lunenfeld, B., Morales, A., Morley, J.E., Schulman, C., Thompson, I.M., Weidner, W. and Wu, F.C.W. (2009) Review ISA, ISSAM, EAU, EAA and ASA Recommendations: Investigation, Treatment and Monitoring of Late-Onset Hypogonadism in Males. International Journal of Impotence Research, 21, 1-8. http://dx.doi.org/10.1038/ijir.2008.41

[7] Castela, A., Vendeira, P. and Costa, C. (2011) Testosterone, Endothelial Health, and Erectile Function. ISRN Endocrinology, 7. http://www.ncbi.nlm.nih.gov/pubmed/22363891

[8] Sauver, J.L.St., Jacobson, D.J., McGree, M.E., Girman, C.J., Klee, G.G., Lieber, M.M. and Jacobsen, S.J. (2011) Associations between Longitudinal Changes in Serum Estrogen, Testosterone, and Bioavailable Testosterone and Changes in Benign Urologic Outcomes. American Journal of Epidemiology, 173, 787-796. http://dx.doi.org/10.1093/aje/kwq438

[9] Grossman, M. and Wu, F.C. (2014) Male Androgen Deficiency: A Multisystem Syndrome. Asian Journal of Andrology, 16, 159-160. http://www.asiaandro.com/Abstract.asp?doi=10.4103/1008-682X.122587 http://dx.doi.org/10.4103/1008-682X.122587

[10] Kelly, D.M. and Jones, T.H. (2013) Testosterone: A Metabolic Hormone in Health and Disease. Journal of Endocrinology, 217, 25-45. http://dx.doi.org/10.1530/JOE-12-0455

[11] Kupelian, V., McVary, K.T., Kaplan, S.T., Hall, S.A., Link, C.L., Aiyer, L.P., Mollon, P., Tamimi, N., Rosen, R.C. and McKinlay, J.B. (2013) Association of Lower Urinary Tract Symptoms and the Metabolic Syndrome: Results From the Boston Area Community Health Survey. The Journal of Urology, 189, 107-116.

[12] Gorbachinsky, I., Akpinar, H. and Assimos, D.G. (2010) Metabolic Syndrome and Urologic Diseases. Reviews in Urology, 12, 157-180.

[13] Jang, M. and Berry, D. (2011) Overweight, Obesity and Metabolic Syndrome in Adults and Children in South Korea: A Review of the Literature. Clinical Nursing Research, 20, 276-291.

[14] Atabek, M.E., Eklioglu, B.S. and Akyurek, N. (2013) Reevaluation of the Prevalence of Metabolic Syndrome in an Urban Area of Turkey. Journal of Clinical Research in Pediatric Endocrinology, 5, 50-54.

[15] Heinonen, I., Helajiarvi, H., Pahkala, K., Heinonen, O.J., Hirvensalo, M., Palve, K., Tammelin, T., Yang, X., Joonala, M., Mikkila, V., Kahonen, M., Lehtimaki, T., Viikari, J. and Raitakari, O.T. (2013) Sedentary Behaviors and Obesity in Adults: The Cardiovascular Risk in Young Finns Study. BMJ Open, 3. http://www.ncbi.nlm.nih.gov/pubmed/23794543

[16] Alberti, K.G.M.M., Eckel, R.H., Grundy, S.M., Zimmet, P.Z., Cleeman, J.I., Donato, K.A., Fruchart, J.-Ch., James, W.P.T., Loria, C.M. and Smith, S.C. (2009) Harmonizing the Metabolic Syndrome: A Joint Interim Statement of the International Diabetes Federation Task Force on Epidemiology and Prevention; National Heart, Lung and Blood Institute; American Heart Association; World Heart Federation; International Atherosclerosis Society and International Association for the Study of Obesity. Circulation, 120, 1640-1645. http://dx.doi.org/10.1161/CIRCULATIONAHA.109.192644

[17] Barry, M.J., Fowler Jr., F.J., O’Leary, M.P., Bruskewitz, R.C., Holtgrewe, H.L., Mebust, W.K. and Cockett, A.T. (1992) The American Urological Association Symptom Index for Benign Prostatic Hyperplasia. The Measurement Committee of the American Urological Association. The Journal of Urology, 148, 1549-1557.

[18] Rosen, R.C., Riley, A., Wagner, G., Osterloh, I.H., Kirkpatrick, J. and Mishra, A. (1997) The International Index of Erectile Function (IIEF): A Multidimensional Scale for Assessment of Erectile Dysfunction. Urology, 6, 822-830. http://dx.doi.org/10.1016/S0090-4295(97)00238-0

[19] Mirzaei, M.R., Amini, M. and Aminorroaya, A. (2012) The Prevalence of Hypogonadism in Diabetic Men in Isfahan Endocrine and Metabolism Research Center, Isfahan, Iran. Journal of Research in Medical Sciences, 17, 602-606.

[20] Heinemann, L.A. (2005) Aging Males’ Symptoms Scale: A Standardized Instrument for the Practice. Journal of Endocrinological Investigation, 28, 34-38.

[21] Cheserek, M.J., Wu, G.-R., Shen, L.-Y., Shi, Y.-H. and Le, G.-W. (2014) Disparities in the Prevalence of Metabolic Syndrome (MS) and Its Components among University Employees by Age, Gender and Occupation. Journal of Clinical and Diagnostic Research, 8, 65-69.

[22] Bal, K., Oder, M., Sahin, A.S., Karatas, C.T., Demir, O., Can, E., Gumus, B.H., Ozer, K., Sahin, O. and Esen, A.A. (2007) Prevalence of Metabolic Syndrome and Its Association with Erectile Dysfunction among Urologic Patients: Metabolic Backgrounds of Erectile Dysfunction. Urology, 69, 356-360.

[23] Pinkhasov, B.B., Selyatitskaya, V.G., Karapetyan, A.R. and Astrakhantseva, E.L. (2012) Metabolic Syndrome in Men and Women with Upper or Lower Types of Body Fat Distribution. Health, 4, 1381-1389.

[24] Matsuzawa, Y. (2008) The Role of Fat Topology in the Risk of Disease. International Journal of Obesity, 32, 83-92. 
http://dx.doi.org/10.1038/ijo.2008.243

[25] Loprinzi, P.D., Smit, E. and Mahoney, S. (2014) Physical Activity and Dietary Behavior in US Adults and Their Combined Influence on Health. Mayo Clinic Proceedings, 89, 190-198.

[26] Pan, Y. and Pratt, C.A. (2008) Metabolic Syndrome and Its Association with Diet and Physical Activity in US Adolescents. Journal of the American Dietetic Association, 108, 276-286.

[27] Li, C., Ford, E.S., Li, B., Giles, W.H. and Liu, S. (2010) Association of Testosterone and Sex Hormone-Binding Globulin with Metabolic Syndrome and Insulin Resistance in Men. Diabetes Care, 33, 1618-1624. http://dx.doi.org/10.2337/dc09-1788

[28] Hackett, G., Kirby, M. and Sinclair, A.J. (2014) Testosterone Deficiency, Cardiac Health and Older Men. International Journal of Endocrinology, 10, 1-10.

[29] Chen, I.H., Tsai, Y.S. and Tong, Y.C. (2012) Correlations among Cardiovascular Risk Factors, Prostate Blood Flow and Prostate Volume in Patients with Clinical Benign Prostatic Hyperplasia. Urology, 79, 409-414. http://dx.doi.org/10.1016/j.urology.2011.09.039

[30] Rao, D.P., Dai, S., Lagace, C. and Krewski, D. (2014) Metabolic Syndrome and Chronic Disease. Chronic Diseases and Injuries in Canada, 34, 36-45.

[31] Albersen, M., Orabi, H. and Lue, T.F. (2012) Evaluation and Treatment of Erectile Dysfunction in the Aging Male: A Mini-Review. Gerontology, 58, 3-14.

[32] Corona, G., Rastrelli, G., Monami, M., Saad, F., Luconi, M., Lucchese, M., Facchiano, E., Sforza, A., Forti, G., Mannucci, E. and Maggi, M. (2013) Body Weight Loss Reverts Obesity-Associated Hypogonadotropic Hypogonadism: A Systematic Review and Meta-Analysis. European Journal of Endocrinology, 168, 829-843.

[33] Fui, M.N.T., Dupuis, P. and Grossmann, M. (2014) Lowered Testosterone in Male Obesity: Mechanisms, Morbidity and Management. Asian Journal of Andrology, 16, 223-231. http://dx.doi.org/10.4103/1008-682X.122365

[34] Corona, G., Mannucci, E., Forti, G. and Maggi, M. (2009) Hypogonadism, ED, Metabolic Syndrome and Obesity: A Pathological Link Supporting Cardiovascular Diseases. International Journal of Andrology, 32, 587-598.

[35] Huhtaniemi, I. (2014) Late-Onset Hypogonadism: Current Concepts and Controversies of Pathogenesis, Diagnosis and Treatment. Asian Journal of Andrology, 16, 192-202. http://dx.doi.org/10.4103/1008-682X.122336

[36] Guay, A.T. (2009) The Emerging Link between Hypogonadism and Metabolic Syndrome. Journal of Andrology, 30, 370-376.

\section{Abbreviations}

ED: Erectile Dysfunction

FSH: Follicle-Stimulating Hormone

IIEF: International Index of Erectile Function

IPSS: International Prostate Symptom Score

IPSS-QoL: IPSS-Quality of Life

LH: Luteinizing Hormone

LOH: Late-Onset Hypogonadism

LUTSs: Lower Urinary Tract Symptoms

MetS: Metabolic Syndrome

PSA: Prostate-Specific Antigen

SHBG: Sex Hormone-Binding Globulin

TSH: Thyroid-Stimulating Hormone

TT: Total Testosterone 
Scientific Research Publishing (SCIRP) is one of the largest Open Access journal publishers. It is currently publishing more than 200 open access, online, peer-reviewed journals covering a wide range of academic disciplines. SCIRP serves the worldwide academic communities and contributes to the progress and application of science with its publication.

Other selected journals from SCIRP are listed as below. Submit your manuscript to us via either submit@scirp.org or Online Submission Portal.
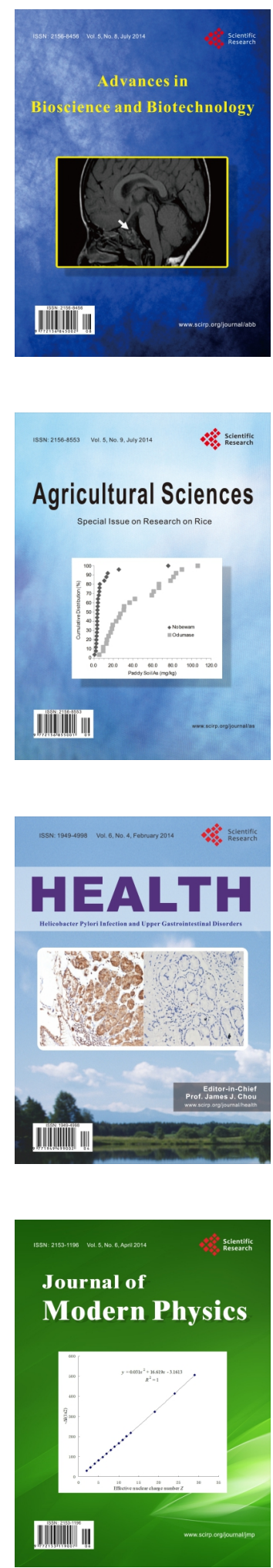
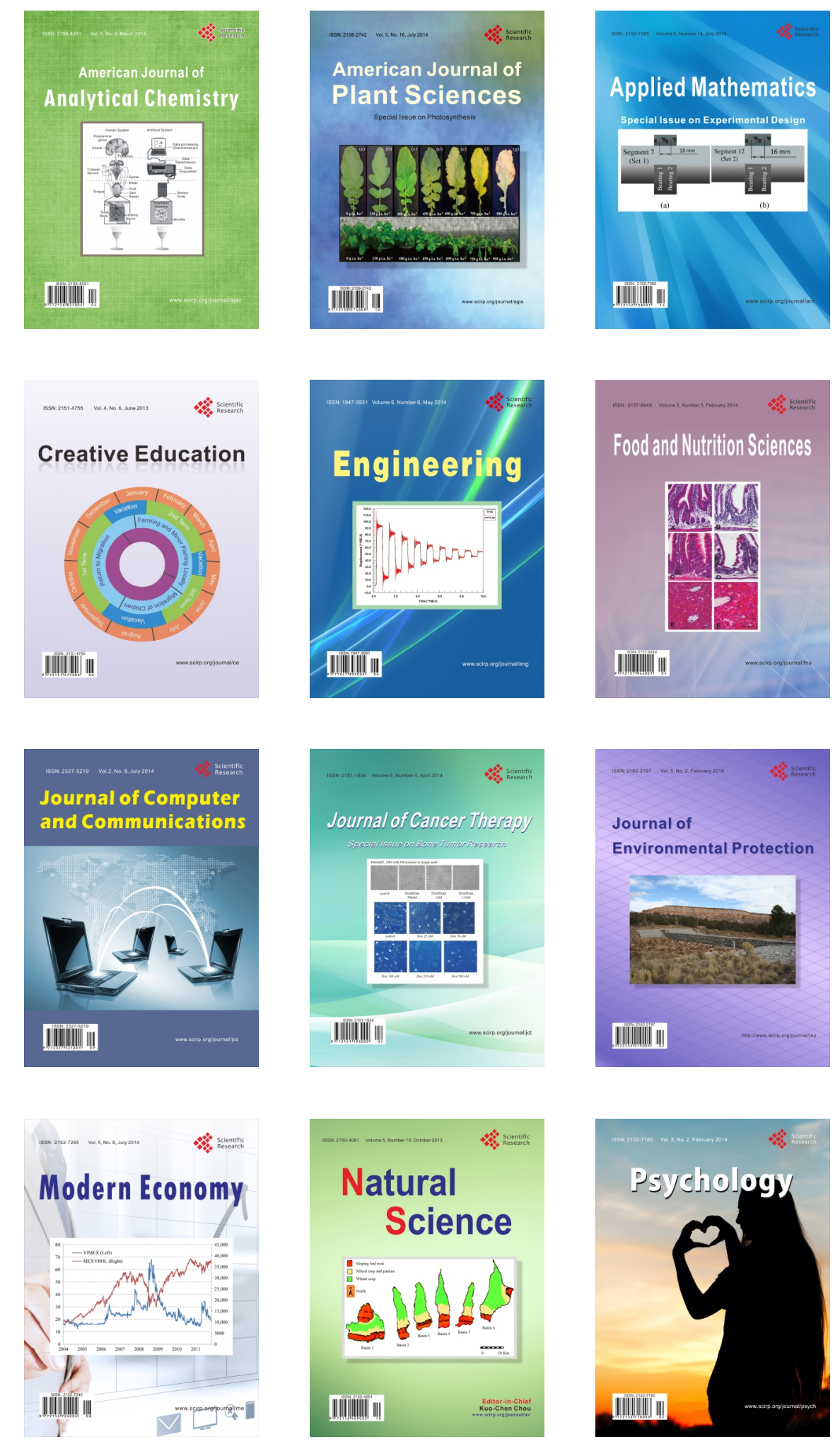